Personal View Article

\title{
Reflective Practice in Anesthesia Clinical Teaching
}

\author{
AssumptaYamuragiye ${ }^{1^{*}, 2}$, Elizabeth Anne Kinsella ${ }^{2}$ \\ ${ }^{1}$ School of Health sciences, College of Medicine and Health Sciences, University of Rwanda, Kigali, Rwanda \\ ${ }^{2}$ Health and Rehabilitation Sciences, Faculty of Health Sciences, University of Western Ontario London, Ontario, Canada
}

*Corresponding author: Assumpta Yamuragiye. School of Health sciences, College of Medicine and Health Sciences, University of Rwanda, Kigali, Rwanda. Email: assoumpta1@gmail.com

\begin{abstract}
\section{Background}

Reflective practice is an essential aspect of knowledge generation for professional practice. By reflecting on action, professionals learn to improve their practices. Through processes of reflection, practitioners participate in a dialogue between theory and practice. Even though reflective practice is an important approach for learning from experience, its place remains unclear in anesthesia clinical education as well as anesthesia practice in a broad sense.
\end{abstract}

\section{Aim}

The aim of this paper was to examine the affordances of reflective practice in anesthesia clinical education.

\section{Methods}

Two cases, illustrating critical incidents in the anesthesia clinical teaching environment, were examined to consider how incorporating reflective practice into clinical education can advance knowledge generation in the field.

\section{Findings}

The two cases studies show how reflective practice can contribute to experiential learning, particularly through reflection on critical incidents.

\section{Conclusion}

Reflective practice can help bridge the gap between theoretical knowledge and practice in anesthesia education and practice.

Rwanda J Med Health Sci 2021;4(3):406-411

Keywords: Reflective practice, anesthesia, clinical teaching, critical incidents, experiential learning

\section{Introduction}

Anesthesia can be defined as a state of reversible loss of awareness and sensation induced by anesthetic drugs. [1] It may include muscle paralysis, analgesia and amnesia.[1] Like other medical disciplines, anesthesia requires disciplinary knowledge and clinical skills, which can be obtained through effective clinical teaching.[2] Anesthesia services are primarily provided by two types of practitioners: physician anesthesiologists and non-physician anesthetists.[1,3] Physician anesthesiologists are medical doctors who have completed a recognized residency in anesthesia specialty.[3]
Non-physician anesthetists include anesthesiologist assistants, certified nurse anesthetists, anesthetist assistants, anesthesia practitioners, anesthesia clinical officers, and anesthesia technicians, among others.[1]

The clinical education in each of these professions varies from one country to another depending on the specific context's expectations.[2] However, what is common across clinical preparation in the anesthesiology field is education oriented toward a knowledge base in anatomy, physiology, pharmacology, critical care, pathophysiology and various anesthesia techniques. [2,3] 
Non-anesthesia technical domains such as communication, leadership and ethics are also taught. [4] Learners in anesthesia rotate through different departments such as emergency, operating theatres, intensive care units or other acute care settings to obtain experience working with various clinical conditions.[5, 3] Clinical supervisors in anesthesiology teach students how to perform anesthetic procedures. [1] In some contexts, clinical supervisors set out a number of procedures to be performed to judge whether students pass or fail.[2] Different modes of teaching are used in anesthesia and these include (but are not limited to) simulation, case studies, problem-based learning, and practice in the operating theatre. [4] Multiple evaluation methods such as written exams, case presentations, and observation of students' interactions with patients are used to assess anesthesia clinical teaching performance . [4] Evaluation aims to promote learning and provide feedback to enable improvement in students' performance of anesthesia skills and procedures.[4]

In every profession, there is a concern with how professional knowledge is generated.[4, 5] According to Schön, [7] the seminal theorist who coined the term 'reflective practice', people come to understand their practices by thinking about them through processes of reflection. By reflecting on actions, professionals learn to improve their practices. [6] Through the reflective process, practitioners participate in a dialogue between theory and practice.[6] The reflective process is therefore a thoughtful way to mediate knowledge talking to action, as well as theory talking to practice.[6,9] Even though reflective practice is an important approach for learning from experience, its place remains unclear in anesthesia clinical education as well as anesthesia practice. $[8,9]$ Anesthesia is a complex medical subspecialty full of critical incidents. [2] Critical incidents frequently stimulate reflection, suggesting that reflective practice could play a significant role in education and learning in this context. Unfortunately, there is limited extant literature focused on reflective practice in anesthesia clinical education or anesthesia practice. The aim of this paper is to examine the affordances of reflective practice to promote learning in anesthesia clinical education, by examining two clinical cases involving critical incidents. The paper draws on seminal literature on reflective practice.

\section{Reflective practice}

Reflective practice is difficult to conceptualize. It is often presented as a simple judgment rather than a philosophical concept.[10] Reflective practice is defined it as "a dialogue of thinking and doing through which I become more skillful". [10] Reflective practice is a complex philosophical concept that involves ongoing reflective thinking, analysis of outcomes, and learning from experience, leading to improvements in performance. It is an important concept in the education process. [8]

In traditional views, reflection occurs retrospectively after an action has taken place. [8] This conception has been extended to consider reflection as also occurring in the midst of action.[10,11] Reflection-on-action occurs after an event, for instance an anesthetist might reflect back following a procedure and think about what they did, what they learned and what they will do next time in a similar situation. Reflection-in-action occurs in the midst of a situation, whereby the anesthetist makes changes to what they are doing in the midst of a procedure as they reflect in the moment on what is unfolding in the situation. Other reflective practice theorists have pointed out a third type of reflection that occurs before an action occurs; anticipatory reflection can be used to plan ahead and be applied to anesthesia clinical education where instructors and students elaborate the anesthesia plan before initiating the anesthesia practice.[2]

There is a great opportunity for reflective practice in health professional education as learners and instructors are exposed to handson practice and opportunities to link theory to practice and learn from experience to improve performance.[12] Anesthesia clinical education offers students a chance to strengthen their knowledge and apply it to real patient care conditions.[1] Through the process of reflecting in and on action, the student acquires professional and personal capabilities relevant for practice.[2] Clinical supervision in medical fields can encourage reflection to enable students to regularly reflect on their practice to deepen learning, and to develop personalized ongoing education plans.[13] Recognizing reflection as a way to improve clinical practice raises questions about how reflective practice can be integrated into anesthesia clinical teaching. 


\section{Reflective practice and anesthesia clinical teaching}

Teaching and learning in a clinical environment require availability of sufficient clinical cases to allow students opportunities to translate theoretical knowledge to practice.[2] Within clinical education, both instructors and students require reflective capabilities to respond to clinical scenarios effectively, contribute to quality healthcare, and advance the process of knowledge generation.[14] Through clinical practice, students move from propositional knowledge or "knowing-that" to performancebased knowledge or "knowing-how".[15] In the process of learning by doing, learners apply their theoretical knowledge into practice.[15] Thus, clinical education allows learners in the medical and allied health professions fields to move from novice to expert practice over time. [15] Practitioners acquire knowledge through reflection on their experience, and learn to negotiate challenges emerging in professional practice.[12] The clinical site is both a place to apply theory into practice and a site for developing knowledge.[16] It is common in anesthesia clinical teaching to face unexpected events which require individual responses. [2] These uncertainties are referred to as the "indeterminate zones" of practice where a problematic situation is uncertain, and therefore the technical problem solving depends on prior construction of a well-formed problem.[10] When a practitioner recognizes a unique situation, she or he cannot handle it solely by applying theories or professional knowledge delivered from her store of professional knowledge.[10] Rather, the indeterminate situations require a dialogue of thinking and imagination to first "set" the problem, and then to search for an appropriate solution to a problem at hand.

\section{Integrating reflective practice into anesthesia clinical education.}

It is common in anesthesia clinical education to debrief and provide feedback about the procedure performed; supervisors and students usually meet to discuss cases and to identify what went well and what could be improved in the student's performance and management of the case.[4] This process of feedback, however, does not generally occur in a systematic manner, but rather occurs on an ad hoc basis, often in response to a dramatic incident that requires discussion. Regular opportunities for collaborative reflection between learners and instructors could allow sufficient time for deeper reflection and advance students' professional knowledge generation.
Ongoing reflection in anesthesia clinical practice offers an opportunity for students to learn from the experience and plan for improvement. Reflection-on-action, whereby thinking about an event after it occurs, questioning oneself about what went well and what went wrong, and thinking about what could have been done in a better or different way, can foster learning and improvement in future performance [10]. Reflection-on-action could be encouraged in anesthesia clinical education as a means to foster the learning process.

In anesthesia practice, there are numerous options to choose from when anesthetizing a patient.[1] The choice depends on the knowledge of the practitioner, the patient's clinical condition, the availability of resources, and in some situations the patient's choice.[1] This process requires a practitioner to constantly think, judge, and use previous experience to determine the best course of action.[4] The subsequent action precipitates another experience that will again refine one's practice-based knowledge for the future. Ongoing reflection on the different aspects of anesthesia practice has the potential to be a source of professional learning and a means of developing practice-based knowledge. [4]

Checklists are used in anesthesia practice to try to standardize the practice, and in some cases to guide learners and novice practitioners.[14] Checklists guide students and practitioners in the equipment required for particular procedures, and in the steps of how the anesthetic procedure is performed in the standard case.[14] Although checklists are helpful in preparing for the standard case, they are not always applicable in challenging situations and indeterminate zones of practice. Reflective processes have the potential to inform practice in such situations.[8] Evaluation tools are used to assess anesthesia students' performance, yet in many circumstances standardized tools are not applicable to the specific situation that arises spontaneously in clinical education. $[6,9,11]$ These constitute situations that require reflection to question assumptions about these tools and their applicability in particular or unique contexts, and to discover solutions based on experiential learning. $[9,11]$ Reflective practice could be encouraged to support learners and clinical instructors in the anesthesia profession to critically think, judge, and analyze the practice to learn from experience and improve future performance.[2] 
Logbooks are used in some settings to document the procedures performed. These could serve as practical pedagogical approaches to engage in reflective practice. Students complete and submit logbooks to supervisors, record their performance, reflect on actions, and develop their knowledge.[16] However, in most cases, these logbooks are used to judge whether the number of cases performed by the student is sufficient for the student to pass, repeat, or fail.[17] The logbooks could in addition be used to encourage reflective learning from experience and knowledge generation.[16] Promoting reflective writing in professional practice could enable students to deepen their understanding, make sense of their own practices, and better grasp complex clinical situations.[6]

Encouraging students to reflect on their own practice through regular reflective writing can speed up the process of integrating theory and evidence into clinical practice.[6] Reflective writing in professional education and practice can take many forms such as reflective journal entries, reflective logs, reflection on critical incidents, case reflections, reflective vignettes, and responses to guided reflection questions, [12] These approaches may assist anesthesia students to reflect on their experience and integrate theory and practice. Reflective writing can also serve as a foundation for collaborative dialogue between students and clinical supervisors, whereby supervisors can 'see' how anesthesia learners are making sense of their clinical and educational experiences.

In the following section two clinical scenarios involving critical incidents are presented as a means to examine the relevance of reflective practice to anesthesia clinical teaching and education.The cases below are illustrative of anesthetist trainees' and practitioners' encounters with such indeterminate situations in practice, and the reflective practice that ensued.

\section{Clinical Scenario 1}

As an example, the first author witnessed the following clinical scenario in anesthesia clinical teaching.

During a surgical procedure, when a student was administering the anesthetic ketamine, and being evaluated by their clinical instructor, a patient failed to fall asleep under the theoretical dose. The clinical instructor intervened and made the decision, along with the team, to continue increasing the dose until the narcosis was obtained. to ketamine,[1] and many of the team members had never experienced this scenario in practice or were unaware that it could occur. As discussed in reflective practice scholarship:

In practice, reflection often begins when a routine response produces a surprise, an unexpected outcome, pleasant or unpleasant. The surprise gets our attention. When intuitive, spontaneous performance yields expected results, then we tend not to think about it; however, when it leads to surprise, we may begin a process of reflection.[18]

In this critical incident, reflection-in-action and reflection-on-action informed the team's decision to a) take over the case from the student, b) increase the dose above the theoretical dose and c) to realize that some patients may require higher doses than expected. While increasing the dose the team was careful to avoid harm to the patient by keeping in mind the lethal dose of ketamine, and by monitoring the patient's vital signs and response, and the rest of the anesthetic management was successful. This is an example of experiential learning generating practice-based knowledge in anesthesia clinical education. Merely following rules and regulations is not sufficient to practice effectively in some situations.[19] There is a need to have an open mind, and to engage in reflection, to determine what is necessary to deal with the problem at hand.

Given that the student was being evaluated, a further issue arose related to the evaluation process and how to award marks. If the evaluation protocol was strictly adhered to, the student would have failed because they would have followed guidelines for ketamine administration according to the theoretical dose and been unsuccessful in the goal of getting the patient to fall asleep, even though no mistake on her part was made, and important learning occurred. The instructor used reflection to contextualize the situation, use professional judgment, and to disregard some evaluation criteria such as effective calculation of hypnotic doses, and successfully anesthetize the patient, to consider the applicable evaluation criteria.

\section{Clinical Scenario 2}

It is not customary for reflective practice to be part of the culture of anesthesia clinical education, especially when the team encounters a complex and stressful clinical scenario.[2] As an example, the first author was involved in anesthesia clinical supervision and encountered a patient with total spinal anesthesia who was undergoing a caesarian section. In this highly stressful emergency situation, senior staff were called to help the anesthesia team manage the patient. 
The entire team was mobilized to resuscitate both the patient and the baby. In anesthesia there exist theories for management of total spinal anesthesia, which can be learned without fully understanding the implications until one witness them in practice. While an explication of theories of total spinal anesthesia is beyond the scope of this paper, one example is a need for rapid tracheal intubation to support ventilation as the patient is totally paralyzed and experiences apnea following a regional anesthesia.

Theories of reflective practice point out that there is a kind of knowledge that is distinct from the propositional knowledge presented in the books and scientific papers, a knowledge embedded in the professional practice itself. $[6,10]$ In this clinical scenario, we examine the critical incident as it relates to the student's learning and reflection. As this became a severe case requiring expert intervention, the student who was initially involved had no further role to play. Nonetheless, the student was present and able to observe the team working with the patient. After successfully resuscitating the patient and the baby, there was not time for further discussion or feedback to the student. Due to these factors, there was no opportunity in the moment to check whether the student had understood the complex management that had occurred, or to optimize this opportunity in the moment for the student to learn how to manage spinal anesthesia. Feedback and dialogue are frequently used approaches to guide learners to reflect on their practices and to cultivate knowledge that informs subsequent improvements in performance.

In anesthesia clinical practice there is frequently insufficient time for such feedback especially when the practice involves difficult clinical scenarios.[17] Although, total spinal anesthesia is an infrequent but serious complication, when it occurs in clinical teaching, it could provide an opportunity for learners to understand its complex management in a real-life practice situation. The following day, the instructor met with the student to debrief about the situation and was surprised that the student's logbook notes were composed as if nothing critical had happened. Despite the stressful event that occurred, the student did not appear to recognize the severity of the situation. Furthermore, the logbook did not offer sufficient space or format for the student to describe the scenario and his reflections. The student's lack of response toward a critical situation that should have galvanized his attention, suggests that opportunities to engage in reflection and feedback should be systematically built into clinical education. Furthermore, logbooks could better be designed to allow for reflective thought and integration of learning from practice experience. A study conducted by Wong,[18] illustrated the power of reflective writing to foster student reflection and knowledge generation in anesthesia education. Perhaps had reflective practice, reflection on critical incidents, reflective writing, and collaborative reflection been introduced into the clinical education earlier, this student would have had opportunities to examine critical scenarios in practice, and to reflect, analyze, and learn from such experiences in the moment. Fortunately, in this situation, the educator was able to work with the student retrospectively to facilitate the required learning, and to reflect on the situation and the implications for education of future students.

\section{Conclusion}

The two clinical scenarios show how reflective practice can be an important aspect of learning through reflection on experience in clinical anesthesia education. Where there is no reflection, a gap between theoretical knowledge and practice prevails.[17] Anesthesia clinical education involves learners and instructors in the application of theoretical knowledge and evidence into anesthesia practice. Through exposure to complex clinical scenarios practitioners use reflective processes to make judgments and handle cases effectively. The anesthesia choice depends on the practitioner's knowledge, availability of resources, the patient's medical profile, and in some situations, the choice of the patient. Therefore, there is a constant need for reflection to plan the anesthesia practice intervention, and to respond effectively to the complex clinical scenarios that arise spontaneously in clinical practice. The clinical field of anesthesia practice should be regarded as a place to apply theory and evidence into practice and as a site for knowledge generation. As such, reflective practice is an important dimension of developing professional knowledge. Nonetheless, its place in anesthesia clinical education and the anesthesia profession has not yet been acknowledged or realized. In addition, the promise and potential of collaborative forms of reflection have yet to be explored in this domain. Reflective writing is a process that could be encouraged in anesthesia clinical teaching to help students to explore complex clinical scenarios, to improve clinical performance, and to foster communication with instructors. Even though logbooks, case studies, and 
checklists, are used in anesthesia clinical education, further reflective writing strategies could be adopted to systematically engage students and instructors in reflective practice to advance the process of knowledge generation. Collaborative reflection, which is an effective way to help students learn from challenges, and build on their positive performance, should become a systematic dimension of anesthesia clinical education. Despite the relevance of reflective practice there is a challenge to find time for reflection in clinical anesthesia education, especially when managing complex and challenging clinical situations. The organizational culture could advance the field by encouraging reflective practice in clinical teaching.

\section{Author's contribution}

AY contributed in conceptualizing ideas, illustrating the clinical scenarios, methodology, conducting a literature review, and writing the manuscript. Then AEK was mainly through editorial support and crafting the manuscript for publication.

\section{Conflict of interest}

We have no conflicts of interest to disclose.

This article is published open access under the Creative Commons Attribution-NonCommercial NoDerivatives (CC BYNC-ND4.0). People can copy and redistribute the article only for noncommercial purposes and as long as they give appropriate credit to the authors. They cannot distribute any modified material obtained by remixing, transforming or building upon this article. See https://creativecommons. org/licenses/by-nc-nd/4.0/

\section{References}

1. Lorraine M. Sdrales RDM. Miller's Anesthesia Review. second 2nd ed. Anesth. Analg. United States: Saunders Elsevier, 2013.

2. Wong A. Review article: Teaching, learning, and the pursuit of excellence in anesthesia education. Can J Anesth. 2012;59:171-81.

3. Gelb AW, Morriss WW, Johnson W, Merry AF. World Health Organization-World Federation of Societies of Anaesthesiologists (WHO-WFSA) International Standards for a Safe Practice of Anesthesia. Can J Anesth Can d'anesthésie. 2018;65:698-708.

4. Frost EAM. Comprehensive Guide to Education in Anesthesia. Springer, 2013.

5. Kemmis S. Knowing practice: searching for saliences. Pedagog Cult Soc. 2005;13:391-426.

6. Ng SL, Kinsella EA, Friesen F, Hodges B. Reclaiming a theoretical orientation to reflection in medical education research: A critical narrative review. Med Educ. 2015;49:461-75.
7. Schön D. Teaching artistry through reflection-in-action. Educating the reflective practitioner. 1987.

8. Kinsella EA. Embodied reflection and the epistemology of reflective practice. $J$ Philos Educ. 2007;41:395-409.

9. Kinsella EA. The art of reflective practice in health and social care: Reflections on the legacy of Donald Schön. Reflective Pract. 2010;11:565-75.

10.Kinsella EA. Professional knowledge and the epistemology of reflective practice. Nurs Philos. 2010;11:3-14.

11. Schön DA. The Reflective Practitioner. Basic book. Reflective Pract. How Prof. Think Action. Routledge; 2017.

12.Larsen DP, London DA, Emke AR. Using reflection to influence practice: student perceptions of daily reflection in clinical education. Perspect Med Educ. 2016;5:28591.

13. Fowler J, Chevannes M. Evaluating the efficacy of reflective practice within the context of clinical supervision. J Adv Nurs. 1998;27:379-82.

14. Chilkoti G, Mohta M, Wadhwa R, Saxena AK. Problem-Based Learning Research in Anesthesia Teaching: Current Status and Future Perspective. Anesthesiol Res Pract. 2014;2014:1-7.

15.Dreyfus. Five Steps from Novice to Expert. Oxford: Basil Blackwell; 1986.

16. Schüttpelz-Brauns K, Narciss E, Schneyinck C, Böhme K, Brüstle P, Mau-Holzmann U, et al. Twelve tips for successfully implementing logbooks in clinical training. Med Teach. 2016;38:564-9.

17. Alhaqwi AI, Taha WS. Promoting excellence in teaching and learning in clinical education. J Taibah Univ Med Sci. Elsevier Ltd; 2015;10:97-101.

18.Wong A, Trollope-Kumar K. Reflections: An inquiry into medical students' professional identity formation. Med Educ. 2014;48:489501. 